\title{
An Improved Possibilistic C-Means Algorithm Based on Kernel Methods
}

\author{
Xiao-Hong $\mathrm{Wu}^{1,2}$ and Jian-Jiang Zhou ${ }^{1}$ \\ ${ }^{1}$ College of Information Science \& Technology, Nanjing University of Aeronautics \\ and Astronautics, Nanjing, 210016, China \\ wxhong@nuaa.edu.cn \\ ${ }^{2}$ College of Electrical \& Information Engineering, Jiangsu University, \\ Zhenjiang, 212013, China
}

\begin{abstract}
A novel fuzzy clustering algorithm, called kernel improved possibilistic c-means (KIPCM) algorithm, is presented based on kernel methods. KIPCM is an extension of the improved possibilistic c-means (IPCM) algorithm. Different from IPCM which is applied in Euclidean space, KIPCM can make data clustering in kernel feature space. With kernel methods the input data can be implicitly mapped into a high-dimensional feature space where the nonlinear pattern now appears linear. It is unnecessary to calculate in this highdimensional feature space because we directly calculate inner products from the input data by kernel function. KIPCM can identify clusters of complex shapes and solve nonlinear separable problems better than IPCM and FCM (fuzzy cmeans). Our experiments show that the proposed algorithm compares favorably with FCM and IPCM.
\end{abstract}

\section{Introduction}

Fuzzy clustering is one of the important unsupervised learning algorithms and fuzzy clustering always has significant advantages over traditional clustering. The wellknown fuzzy clustering is the fuzzy c-means (FCM) algorithm [1]. FCM algorithm makes the memberships of a data point across classes sum to 1 by the probabilistic constraint. And FCM is appropriate to interpret memberships as probabilities of sharing. However, the memberships of FCM do not always correspond to the intuitive concept of degree of belong or compatibility. Furthermore, the FCM is sensitive to noises or outliers [2]. To overcome these disadvantages Krishnapuram and Keller have presented the possibilistic c-means (PCM) algorithm [2] by abandoning the constraint of FCM and constructing a novel objective function. The PCM can cluster noisy data and noisy data have low degrees of compatibility in all clusters, so their effects on the clustering can be neglected. But PCM is very sensitive to good initialization and it has an undesirable tendency to produce coincident clusters [3] that because the columns and rows of the typicality matrix are independent of each other. PCM attaches importance to the notion of typicality that alleviates the undesirable effect of noises but neglects the membership that makes the class centroid close to data points. To overcome the shortcoming of PCM, Zhang and Leung have proposed improved possibilistic c-means (IPCM) algorithm [4]. IPCM solves the noise sensitivity defect of FCM, and also overcomes the coincident clusters problem of PCM. 
However, FCM, PCM and IPCM have the same drawback that they use point prototypes and a norm-induced distance, as a consequence, they obtain good clustering results only when the data set contains clusters of roughly the same size and shape. To identify clusters of various shapes which are complex topological structures in the same data set, kernel methods [5] has been introduced into fuzzy c-means clustering [6]. In this paper we propose kernel improved possibilistic c-means (KIPCM) algorithm based on kernel methods. With kernel methods the input data samples can be mapped implicitly into a high-dimensional feature space where the nonlinear pattern now appears linear and IPCM algorithm is carried out. We need not calculate in highdimensional feature space because the kernel function can do it just in input space.

The rest of this paper is organized as follows: in section 2 the IPCM algorithm is introduced, and in section 3 the KIPCM algorithm is presented. Some tests and conclusions are given in later section.

\section{Improved Possibilistic C-Means Algorithm}

Given an unlabeled data set $\mathbf{X}=\left\{\mathbf{x}_{1}, \mathbf{x}_{2}, \ldots, \mathbf{x}_{\mathbf{n}}\right\} \subset \mathfrak{R}^{p}$, find the partition of $\mathbf{X}$ into $1<\mathrm{c}<\mathrm{n}$ fuzzy subsets by minimizing the following objective function

$$
J_{m}(\mathbf{U}, \mathbf{T}, \mathbf{V})=\sum_{i=1}^{c} \sum_{k=1}^{n} u_{i k}^{m} t_{i k} D_{i k}^{2}+\sum_{i=1}^{c} \eta_{i} \sum_{k=1}^{n} u_{i k}^{m}\left(t_{i k} \log t_{i k}-t_{i k}+1\right)
$$

subject to the constraints: $0 \leq u_{i k}, t_{i k} \leq 1, D_{i k}=\left\|\mathrm{x}_{k}-v_{i}\right\|$, and $\sum_{i=1}^{c} u_{i k}=1, \forall k$. Where $\mathrm{c}$ is the number of clusters and $\mathrm{n}$ is the number of data points, $u_{i k}$ is the membership of $\mathrm{x}_{k}$ in class $i, t_{i k}$ is the possibilistic (typicality) value $\mathrm{x}_{k}$ in class $i$, and $\mathrm{m}$ is a weighting exponent, $\mathrm{m} \in[1, \infty)$.

Then $\min _{(\mathbf{U}, \mathbf{T}, \mathbf{V})} J_{m}(\mathbf{U}, \mathbf{T}, \mathbf{V})$ is optimized under constraints and the following equations are obtained

$$
\begin{gathered}
u_{i k}=\left[\sum_{j=1}^{c}\left(\frac{\eta_{i}\left(1-\exp \left(-\frac{D_{i k}^{2}}{\eta_{i}}\right)\right)}{\eta_{j}\left(1-\exp \left(-\frac{D_{j k}^{2}}{\eta_{j}}\right)\right)}\right)^{\frac{2}{m-1}}\right]^{-1}, \forall i, k \\
t_{i k}=\exp \left(-\frac{D_{i k}^{2}}{\eta_{i}}\right), \forall i, k
\end{gathered}
$$




$$
v_{i}=\frac{\sum_{k=1}^{n} u_{i k}^{m} t_{i k} \mathbf{x}_{k}}{\sum_{k=1}^{n} u_{i k}^{m} t_{i k}}, \forall i
$$

Where $v_{i}$ is the cluster center or prototype of $\mathrm{u}_{i} \cdot \eta_{i}$ in PCM is described as

$$
\eta_{i}=K \frac{\sum_{k=1}^{n} u_{i k}^{m} D_{i k}^{2}}{\sum_{k=1}^{n} u_{i k}^{m}}, K>0
$$

Here, $K$ is always chosen to be 1 in equation (3) and (4).

If $D_{i k}>0$ for all $i$ and $\mathrm{k}, \mathrm{m}>1$, and $\mathbf{X}$ contains $\mathrm{c}<\mathrm{n}$ distinct data points, then the algorithm described below is called IPCM-AO algorithm:

\section{Initialization}

1) Fix $\mathrm{c}, \mathrm{m}$ and $w, 1<\mathrm{c}<\mathrm{n}, 1<\mathrm{m}, w<+\infty$; Set iteration counter $\mathrm{r}=1$ and maximum iteration $r_{\max }$

2) Run FCM until termination to get initial membership $\mathbf{U}^{(0)}$ and initial cluster centers $\boldsymbol{V}^{(0)}$. Then use equation (3) to get $\eta_{i}$.

\section{Repeat}

Step 1 Update typicality matrix $\mathbf{T}^{\mathrm{r}}$ by equation (2b) ;

Step 2 Update membership matrix $\mathbf{U}^{\mathrm{r}}$ by equation (2a);

Step 3 Update $\boldsymbol{V}^{\mathbf{r}}$ by equation (2c);

Step 4 Increment $r$;

Until $\left(\left\|\mathrm{U}^{r}-\mathrm{U}^{r-1}\right\|<\mathcal{E}\right)$ or $\mathrm{r}>\mathrm{r}_{\max }$

\section{Kernel Improved Possibilistic C-Means Algorithm}

With the theory of Mercer kernel [7], the input space $\mathrm{X}$ is mapped into a novel high dimensional feature space $\mathrm{F}$ :

$$
\mathrm{X}=\left(x_{1}, \ldots, x_{M}\right) \rightarrow \Phi(\mathrm{X})=\left(\phi\left(\mathrm{X}_{1}\right), \ldots, \phi\left(\mathrm{X}_{N}\right)\right)
$$

Kernel function K satisfies:

$$
K\left(x_{i}, x_{j}\right)=<\phi\left(x_{i}\right) \cdot \phi\left(x_{j}\right)>
$$

Scalar product calculation in input space is transformed into kernel function calculation by nonlinear mapping: 


$$
<x_{i} \cdot x_{j}>\rightarrow<\phi\left(x_{i}\right) \cdot \phi\left(x_{j}\right)>=K\left(x_{i}, x_{j}\right)
$$

Then the objective function (1) is transformed as follows

$$
\begin{gathered}
J_{m, w}(\mathbf{U}, \mathbf{T}, \mathbf{V})=\sum_{i=1}^{c} \sum_{k=1}^{n} u_{i k}^{m} t_{i k}\left\|\phi\left(\mathrm{x}_{k}\right)-\phi\left(v_{i}\right)\right\|^{2} \\
+\sum_{i=1}^{c} \eta_{i} \sum_{k=1}^{n} u_{i k}^{m}\left(t_{i k} \log t_{i k}-t_{i k}+1\right) \\
\left\|\phi\left(\mathrm{x}_{k}\right)-\phi\left(v_{i}\right)\right\|^{2}=<\left[\phi\left(\mathrm{x}_{k}\right)-\phi\left(v_{i}\right)\right] \cdot\left[\phi\left(\mathrm{x}_{k}\right)-\phi\left(v_{i}\right)\right]> \\
=K\left(\mathrm{x}_{k}, \mathrm{x}_{k}\right)+K\left(v_{i}, v_{i}\right)-2 K\left(\mathrm{x}_{k}, v_{i}\right)
\end{gathered}
$$

In this paper, we use Gaussian kernel function:

$$
K(x, y)=\exp \left(-\frac{\|x-y\|^{2}}{2 \sigma^{2}}\right)
$$

So the equation (8) can be written as

$$
\left\|\phi\left(\mathrm{x}_{k}\right)-\phi\left(v_{i}\right)\right\|^{2}=2-2 K\left(\mathrm{x}_{k}, v_{i}\right)
$$

To minimize equation (7), subject to the constraints $\mathrm{m}>1,0 \leq u_{i k}, t_{i k} \leq 1$, and $\sum_{i=1}^{c} u_{i k}=1, \forall k$, we obtain the following equations

$$
\begin{gathered}
u_{i k}=\left[\sum_{j=1}^{c}\left(\frac{\eta_{i}\left(1-\exp \left(-\frac{2-2 K\left(\mathrm{x}_{k}, v_{i}\right)}{\eta_{i}}\right)\right)}{\eta_{j}\left(1-\exp \left(-\frac{2-2 K\left(\mathrm{x}_{k}, v_{j}\right)}{\eta_{j}}\right)\right)}\right]^{\frac{2}{m-1}}\right]^{-1}, \forall i, k \\
t_{i k}=\exp \left(-\frac{2-2 K\left(\mathrm{x}_{k}, v_{i}\right)}{\eta_{i}}\right), \forall i, k \\
\phi\left(v_{i}\right)=\frac{\sum_{k=1}^{n} u_{i k}^{m} t_{i k} \phi\left(\mathbf{x}_{k}\right)}{\sum_{k=1}^{n} u_{i k}^{m} t_{i k}}, \forall i, j
\end{gathered}
$$


Here, equation (11c) can not be calculated directly, and by multiplying $\phi\left(\mathrm{x}_{j}\right)^{T}$ on the left sides of equation (11c), the following equation is obtained

$$
K\left(\mathbf{x}_{j}, v_{i}\right)=\frac{\sum_{k=1}^{n} u_{i k}^{m} t_{i k} K\left(\mathbf{x}_{k}, \mathbf{x}_{j}\right)}{\sum_{k=1}^{n} u_{i k}^{m} t_{i k}}, \forall i, j
$$

In kernel fuzzy c-means (KFCM) algorithm [6], $K\left(\mathbf{x}_{j}, v_{i}\right)$ is calculated as

$$
K\left(\mathbf{x}_{j}, v_{i}\right)=\frac{\sum_{k=1}^{n} u_{i k}^{m} K\left(\mathbf{x}_{k}, \mathbf{x}_{j}\right)}{\sum_{k=1}^{n} u_{i k}^{m}}, \forall i, j
$$

Using kernel methods to equation (3), they are transformed into equation (13)

$$
\eta_{i}=K \frac{\sum_{k=1}^{n} u_{i k}^{m}\left(2-2 K\left(\mathrm{x}_{k}, v_{i}\right)\right)}{\sum_{k=1}^{n} u_{i k}^{m}}, K>0
$$

If $D_{i k}=\left\|\phi\left(\mathrm{x}_{k}\right)-\phi\left(v_{i}\right)\right\|>0$ for all $i$ and $\mathrm{k} \geqslant 1$, and $\mathbf{X}$ contains $\mathrm{c}<\mathrm{n}$ distinct data points, then the algorithm described below is called KIPCM-AO algorithm:

\section{Initialization}

1) Fix c, $\mathrm{m}$ and $\eta, 1<\mathrm{c}<\mathrm{n}, 1<\mathrm{m}, w<+\infty$. Set iteration counter $\mathrm{r}=1$ and maximum iteration $r_{\max }$.

2) Run FCM until termination to get initial membership $\mathbf{U}^{(0)}$ and initial cluster centers $\boldsymbol{V}^{(0)}$. Then use Eq. (13) to get $\eta_{i}$.

3) Calculate the kernel matrix $K_{\mathrm{vx}}^{(0)}$ with $\mathbf{V}^{(0)}$ by equation (12);

\section{Repeat}

Step 1 Update $\mathbf{T}^{(\mathrm{r})}$ with equation (11b), $K_{\mathrm{vx}}^{(\mathrm{r}-1)}$ and $\eta_{i}$;

Step 2 Update membership $\mathbf{U}^{(\mathrm{r})}$ with equation (11a), $K_{\mathrm{vx}}^{(\mathrm{r}-1)}$ and $\mathbf{T}^{(\mathrm{r})}$;

Step 3 Update $K_{\mathrm{vx}}^{(\mathrm{r})}$ with equation (11d), $\mathbf{U}^{(\mathrm{r})}$ and $\mathbf{T}^{(\mathrm{r})}$;

Step 5 Increment $\mathrm{r}$;

Until $\left(\left\|\mathrm{U}^{(r)}-\mathrm{U}^{(r-1)}\right\|<\varepsilon\right)$ or $\mathrm{r}>\mathrm{r}_{\max }$ 


\section{Experiments}

We first do experiment with $\mathbf{X}_{12}$ which is a two dimensional data set with 11 points whose coordinates are given in Table 1 . The data set $\mathbf{X}_{12}$ comes from N. R. Pal [8] and Figure 1 shows its distribution in coordinates. There are ten points (except $\mathbf{x}_{6}$ and $\mathbf{x}_{12}$ ) form two diamond shaped clusters with five points each on the left and right sides of the $y$ axis. We can see $\mathbf{x}_{6}$ and $\mathbf{x}_{12}$ as noisy points and each has the same distance from the two clusters. The initialization of cluster centers

$$
V_{0}=\left[\begin{array}{ll}
0.08 & 0.36 \\
0.41 & 0.99
\end{array}\right]
$$

Computational condition: $\mathcal{E}=0.00001$, maximum number of iterations $=100 . \mathrm{m}=2.0$, $w=2.0$, the width $\sigma$ of Gaussian kernel function is 10 .

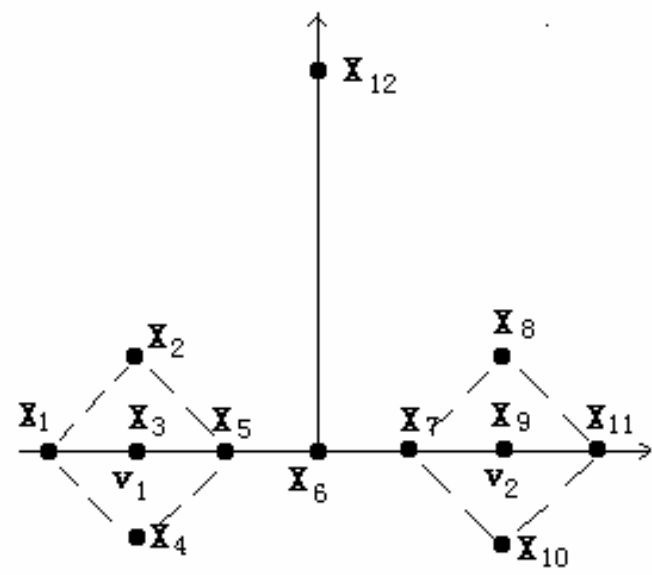

Fig. 1. Data set $\mathbf{X}_{12}$

Table 1 shows the terminal membership values of FCM by applying FCM-AO and Table 2 shows the terminal membership values and typicality values of IPCM by applying IPCM-AO to $\mathbf{X}_{12}$. The memberships of $\mathbf{x}_{6}$ and $\mathbf{x}_{12}$ in both FCM and IPCM are 0.500 in each cluster. From Table 2 IPCM provides both membership and typicality information but FCM provides only membership information. For example, the typicality values of $\mathbf{x}_{6}$ and $\mathbf{x}_{12}$ are 0.27 and 0.00 , that is to say, $\mathbf{x}_{12}$ is more atypical than $\mathbf{x}_{6}$ for either cluster. So IPCM can distinguish between $\mathbf{x}_{6}$ and $\mathbf{x}_{12}$ and distinguish them from other data. FCM considers $\mathbf{x}_{6}$ identical with $\mathbf{x}_{12}$ but it is not the fact. So FCM can not distinguish noises from input data. In conclusion FCM is more sensitive to noises than IPCM.

Table 3 shows the terminal membership values and typicality values of KIPCM by applying KIPCM-AO to $\mathbf{X}_{12}$. Both KIPCM and IPCM can avoid the influence of 
Table 1. Data set $\mathbf{X}_{12}$ and terminal $\mathbf{U}$ from FCM for $\mathbf{X}_{12}$

\begin{tabular}{|c|c|c|c|c|}
\hline & \multicolumn{2}{|c|}{ Data set $\mathbf{X}_{12}$} & \multicolumn{2}{c|}{ FCM } \\
\hline Pt. & $\mathrm{x}$ & $\mathrm{y}$ & $\mathrm{U}_{1}^{\mathrm{T}}$ & $\mathrm{U}_{2}^{\mathrm{T}}$ \\
\hline 1 & -5.00 & 0.00 & 0.94 & 0.06 \\
\hline 2 & -3.34 & 1.67 & 0.97 & 0.03 \\
\hline 3 & -3.34 & 0.00 & 0.99 & 0.01 \\
\hline 4 & -3.34 & -1.67 & 0.90 & 0.10 \\
\hline 5 & -1.67 & 0.00 & 0.92 & 0.08 \\
\hline 6 & 0.00 & 0.00 & 0.50 & 0.50 \\
\hline 7 & 1.67 & 0.00 & 0.08 & 0.92 \\
\hline 8 & 3.34 & 1.67 & 0.03 & 0.97 \\
\hline 9 & 3.34 & 0.00 & 0.01 & 0.99 \\
\hline 10 & 3.34 & -1.67 & 0.10 & 0.90 \\
\hline 11 & 5.00 & 0.00 & 0.06 & 0.94 \\
\hline 12 & 0.00 & 10.00 & 0.50 & 0.50 \\
\hline
\end{tabular}

Table 2. Terminal $\mathbf{U}$ and $\mathbf{T}$ from IPCM for $\mathbf{X}_{12}$

\begin{tabular}{|c|c|c|c|c|}
\hline Pt. & $\mathrm{U}_{1}^{\mathrm{T}}$ & $\mathrm{U}_{2}^{\mathrm{T}}$ & $\mathrm{T}_{1}^{\mathrm{T}}$ & $\mathrm{T}_{2}^{\mathrm{T}}$ \\
\hline 1 & 0.90 & 0.10 & 0.67 & 0.00 \\
\hline 2 & 0.92 & 0.08 & 0.70 & 0.00 \\
\hline 3 & 1.00 & 0.00 & 1.00 & 0.00 \\
\hline 4 & 0.92 & 0.08 & 0.70 & 0.00 \\
\hline 5 & 0.93 & 0.07 & 0.73 & 0.05 \\
\hline 6 & 0.50 & 0.5 & 0.27 & 0.27 \\
\hline 7 & 0.07 & 0.93 & 0.05 & 0.73 \\
\hline 8 & 0.08 & 0.92 & 0.00 & 0.70 \\
\hline 9 & 0.00 & 1.00 & 0.00 & 1.00 \\
\hline 10 & 0.08 & 0.92 & 0.00 & 0.70 \\
\hline 11 & 0.10 & 0.90 & 0.00 & 0.67 \\
\hline 12 & 0.50 & 0.50 & 0.00 & 0.00 \\
\hline
\end{tabular}

noises or outlier. In Table 2 the typicality values of $\mathbf{x}_{6}$ and $\mathbf{x}_{12}$ from KIPCM are smaller than that from IPCM. So KIPCM is more insensitive to noises than IPCM.

The other example is that we perform experiments on IRIS data set [9]. The computational condition is $\mathcal{E}=0.00001$, maximum number of iterations $=100, \mathrm{~m}=2.0$, $w=2.0$, the width $\sigma$ of Gaussian kernel function is 3. The clustering accuracy from FCM, IPCM and KIPCM on IRIS data set is illustrated in Table 4. The KIPCM algorithm has better clustering accuracy than the other two algorithms evidently. 
Table 3. Terminal U and T from KIPCM for $\mathbf{X}_{12}$

\begin{tabular}{|c|c|c|c|c|}
\hline Pt. & $\mathrm{U}_{1}^{\mathrm{T}}$ & $\mathrm{U}_{2}^{\mathrm{T}}$ & $\mathrm{T}_{1}^{\mathrm{T}}$ & $\mathrm{T}_{2}^{\mathrm{T}}$ \\
\hline 1 & 0.78 & 0.22 & 0.47 & 0.00 \\
\hline 2 & 0.80 & 0.20 & 0.50 & 0.00 \\
\hline 3 & 0.94 & 0.06 & 0.74 & 0.00 \\
\hline 4 & 0.80 & 0.20 & 0.50 & 0.00 \\
\hline 5 & 0.81 & 0.19 & 0.53 & 0.03 \\
\hline 6 & 0.50 & 0.50 & 0.17 & 0.17 \\
\hline 7 & 0.19 & 0.81 & 0.03 & 0.53 \\
\hline 8 & 0.20 & 0.80 & 0.00 & 0.50 \\
\hline 9 & 0.06 & 0.94 & 0.00 & 0.74 \\
\hline 10 & 0.20 & 0.80 & 0.00 & 0.50 \\
\hline 11 & 0.22 & 0.78 & 0.00 & 0.47 \\
\hline 12 & 0.50 & 0.50 & 0.00 & 0.00 \\
\hline
\end{tabular}

Table 4. Clustering accuracy from FCM, IPCM and KIPCM for IRIS data set

\begin{tabular}{|c|c|c|c|}
\hline Data set & FCM & IPCM & KIPCM \\
\hline IRIS & $89.3 \%$ & $92.0 \%$ & $93.3 \%$ \\
\hline
\end{tabular}

\section{Conclusions}

Based on kernel methods we propose kernel improved possibilistic c-means (KIPCM) algorithm as an extension of improved possibilistic c-means (IPCM) algorithm. The KIPCM computes both membership and typicality values the same as IPCM. However, KIPCM can map input data points to a high-dimensional feature space where clustering unlabeled data is carried out. By using kernel method the KIPCM can deal with noises or outliers better than IPCM. Furthermore KIPCM can deal with linear non-separable problem better than FCM and IPCM. Experiments show the better performance of KIPCM.

\section{References}

[1] Bezdek, J. C.: Pattern Recognition with Fuzzy Objective Function Algorithms, Plenum Press, New York (1981)

[2] Krishnapuram, R., and Keller, J.: A Possibilistic Approach to Clustering, IEEE Trans. Fuzzy Systems, 1 (2) (1993) 98-110

[3] Barni, M., Cappellini, V., and Mecocci, A.: Comments on "A Possibilistic Approach to Clustering”, IEEE Trans. Fuzzy Systems, 4(3) (1996) 393-396

[4] Zhang, J.-S., Leung, Y.-W.: Improved possibilistic C-means clustering algorithms, IEEE Trans. Fuzzy Systems, 12(2) (2004) 209-217

[5] Vapnik, V.: Statistical Learning Theory. Wiley (1998) 
[6] Girolami, M.,: Mercer kernel based clustering in feature space, IEEE Trans. on Neural Networks, Vol.13, No.13 (2002) pp. 780-784

[7] Aizerman, M., Braverman, E., and Rozonoer, L.: Theoretical foundations of the potential function method in pattern recognition learning. Automation and Remote Control 25: (1964) 821-837

[8] Pal, N. R., Pal, K., and Bezdek, J. C.: A mixed c-means clustering model, Processings of the IEEE Trans. Fuzzy Systems, Spain (1997) pp.11-21

[9] Anderson, E.: The Iris of Gasp Peninsula, Bulletin of American Iris Society, 59 (1935) 2-5 